\title{
Fatores relacionados ao acolhimento com classificação de risco a idosos em unidades de pronto atendimento
}

\author{
Factors related to supportive reception with risk classification of the elderly in emergency facilities
}

Factores relacionados con la acogida de ancianos con clasificación de riesgo en unidades de atención de emergencia

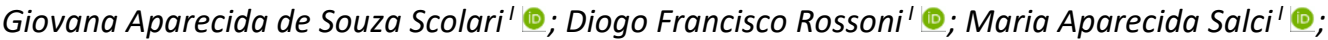 \\ Cremilde Aparecida Trindade Radovanovic' ${ }^{\circledR}$; Lígia Carreira' ${ }^{\circledR}$
}

'Universidade Estadual de Maringá, Maringá, PR, Brasil

\begin{abstract}
RESUMO
Objetivo: analisar o acolhimento com classificação de risco a idosos em unidades de pronto atendimento em um munícipio de médio porte do Sul do país. Método: Estudo transversal e exploratório de abordagem quantitativa. Os dados foram obtidos em prontuários de idosos atendidos em duas unidades de pronto atendimento. A coleta de dados ocorreu no período de janeiro a março de 2016. Foi realizada análise descritiva dos dados utilizando o programa R e o teste Qui-quadrado. Resultados: Foram identificados 2.927 idosos atendidos nesses serviços, a maioria mulheres (55,7\%), entre 60 e 69 anos (48,7\%), o dia da semana com maior número de atendimento foram as segundas-feiras $(18,0 \%)$, com destaque para a classificação verde $(81,6 \%)$. Conclusão: Evidenciou-se alta procura de idosos jovens, sobretudo por condições sensíveis à atenção primária à saúde. Descritores: Acolhimento; Triagem; Enfermagem em Emergência; Idoso.
\end{abstract}

\begin{abstract}
Objective: to examine supportive reception with risk classification for the elderly in emergency facilities in a medium-sized municipality in southern Brazil. Method: in this exploratory, cross-sectional, quantitative study, data were collected, from January to March 2016, from medical records of older adults treated at two emergency facilities. Descriptive analysis of the data used the R program and the Chi-square test. Results: 2,927 elderly people were treated at these services, most of them women (55.7\%), between 60 and 69 years old (48.7\%), most often on Mondays (18.0\%), and classified green (81.6\%). Conclusion: high demand was found from younger elderly, mainly for conditions amenable to primary health care. Descriptors: User Embracement; Triage; Emergency Nursing; Aged.
\end{abstract}

\section{RESUMEN}

Objetivo: analizar la acogida de ancianos, con clasificación de riesgo, en unidades de atención de emergencia de un municipio mediano del sur del país. Método: estudio transversal y exploratorio con enfoque cuantitativo. Los datos se obtuvieron de los registros médicos de los ancianos atendidos en dos unidades de atención de emergencia. La recolección de datos tuvo lugar de enero a marzo de 2016. Se realizó un análisis descriptivo de los datos utilizando el programa $\mathrm{R}$ y la prueba de Chi-cuadrado. Resultados: se identificó que 2.927 ancianos fueron asistidos en estos servicios, la mayoría mujeres (55,7\%), entre 60 y 69 años $(48,7 \%)$, los días de la semana con la mayor cantidad de atención fueron los lunes (18,0\%), con énfasis en la clasificación verde $(81,6 \%)$. Conclusión: se evidenció una gran demanda en cuanto a ancianos más jóvenes, principalmente debido a condiciones sensibles a la atención primaria de salud.

Descriptores: Acogimiento; Triaje; Enfermería de Urgencia; Anciano.

\section{INTRODUÇÃO}

Estimativas apontam que a população idosa mundial passará de 554 milhões em 2013 para aproximadamente 1,6 bilhões até 2050. O fenômeno do envelhecimento populacional tem demonstrado efeitos abrangentes sobre os sistemas sociais, econômicos e principalmente de saúde, pois o crescimento da população está ocorrendo paralelamente às desigualdades de renda, apoio social e lacunas na assistência à saúde devido a padrões complexos da carga de doenças e globalização dos fatores de riscos ${ }^{1}$.

Tais aspectos se intensificam nos países em desenvolvimento, como o Brasil, por contarem com uma vida inteira de riscos à saúde associados à pobreza e ao acesso inadequado aos cuidados de saúde, que tem resultado em idosos com maior incidência e prevalência de Doenças Crônicas Não Transmissíveis (DCNT) ${ }^{1,2}$.

Com a transição epidemiológica, observa-se aumento das agudizações das condições crônicas dos idosos, fazendoos procurar com maior frequência os serviços de saúde, especialmente as unidades emergenciais, que tem se destacado

Agradecimentos à Coordenação de Aperfeiçoamento de Pessoal de Nível Superior (CAPES) - Código de financiamento 001.

Autora correspondente: Giovana Aparecida de Souza Scolari. E-mail: giscolari@hotmail.com

Editora Científica: Cristiane Helena Gallasch; Editora Associada: Sonia Acioli Oliveira 
como porta de entrada dos usuários. Estes motivos podem ser justificados pela modalidade de atenção das emergências, como o gerenciamento dos casos, atendimento 24 horas, com garantia de consulta e acesso a maiores recursos tecnológicos ${ }^{3}$.

No Brasil, o contexto emergencial que tem chamado a atenção dos usuários, são as Unidades de Pronto Atendimento (UPAs) ${ }^{4,5}$. Trata-se de serviços de média complexidade, que tem como objetivo manter a articulação com a Atenção Primária à Saúde (APS), o Serviço de Atendimento Móvel de Urgência (SAMU), a Atenção Domiciliar e a Atenção Hospitalar 6 .

No mesmo ano de implantação das UPAs, o Ministério da Saúde publicou por meio da Política Nacional de Humanização da Atenção e Gestão do SUS, o documento "Acolhimento e Classificação de Risco nos Serviços de Urgência". Consta no texto sobre a melhoria do acesso aos indivíduos, transformando o método tradicional de entrada por filas e ordem de chegada; propondo mudanças na relação entre profissionais e usuários no que diz respeito à forma de escutar seus problemas e demandas, para que a abordagem ao paciente seja além de suas queixas ${ }^{7}$.

Embora a classificação de risco propicie humanização no acolhimento dos indivíduos aos serviços de emergência, sabe-se que alguns fatores interferem em tal prática, a qual tem sido alvo de pesquisas e debates entre gestores ${ }^{8}$. Notase na literatura, algumas pesquisas que abordam a classificação de risco em distintos serviços emergenciais ${ }^{8}$, mas com a pessoa idosa, os estudos são escassos, principalmente no contexto das UPAs. Diante do exposto, questionou-se: qual relação entre a classificação de risco e as variáveis sexo, faixa etária, dias da semana e do mês referentes ao atendimento de idosos em UPAs de um município paranaense?

Ao considerar a importância de sistematizar o acolhimento dos idosos com vistas a proporcionar assistência qualificada em situações emergenciais, julga-se primordial a realização desta pesquisa, cujo objetivo foi analisar o acolhimento com classificação de risco a idosos em unidades de pronto atendimento em um munícipio de médio porte do Sul do país.

\section{MÉTOdo}

Trata-se de um estudo transversal, exploratório, de abordagem quantitativa, desenvolvido nas duas UPAs de um município de médio porte do Noroeste do Paraná (PR).

O município em questão possui o maior índice de idosos entre os oito municípios com mais de 200 mil habitantes do Estado. Atualmente, conta com 397.436 habitantes, destes, 54.190 são idosos, representando 13,6\% da população . Para atender este contingente referente a complexidade intermediária emergencial, os munícipes contam com duas UPAs, ambas inauguradas há sete anos. Para preservar a identidade das instituições, estas serão identificadas como UPA 1 e UPA 2. A UPA 1 é elencada como porte III, com 31 leitos de observação e três de isolamento; a UPA 2 é classificada como porte II, possui uma sala de observação com 12 leitos, incluindo dois leitos de isolamento ${ }^{4}$.

As UPAs funcionam 24 horas por dia, sete dias por semana, sendo que o acolhimento é realizado por enfermeiro, que avalia a queixa principal, os sinais e sintomas, as comorbidades preexistentes, os medicamentos em uso e a prioridade do atendimento. Com essa primeira abordagem, o profissional classifica o risco do atendimento. $O$ protocolo utilizado nas instituições estudadas é baseado nas diretrizes do Ministério da Saúde, e utiliza quatro níveis de classificação de risco: azul (casos não urgentes - pode aguardar até 240 minutos), verde (casos pouco urgentes - pode aguardar até 120 minutos), amarelo (casos urgentes - pode aguardar até 60 minutos) e vermelho (casos de emergência - que requerem atendimento imediato) $)^{7}$.

Como critérios de inclusão, estabeleceu-se que fossem selecionadas todas as fichas de atendimento de pessoas com idade igual ou maior a 60 anos atendidos no mês de novembro de 2015 nas UPAs.

A coleta de dados foi realizada entre os meses de janeiro e março de 2016, utilizando-se um instrumento elaborado pelos pesquisadores, contendo: os dados sociodemográficos (idade e sexo) e os dados de atendimento (dia da semana, dia do mês e a classificação de risco). Foram consideradas variáveis sociodemográficas o sexo e a faixa etária. Os dados de atendimento selecionados foram: dia da semana, dia do mês e classificação de risco. Nesta pesquisa, considerou-se como exposição o atendimento em duas unidades de pronto atendimento, categorizado em UPA 1 e UPA 2 e a variável desfecho foi a classificação de risco dos idosos.

Posteriormente, os dados foram categorizados em uma planilha do Programa software Microsoft Office Excel, versão 2010, e checados para correção de possíveis erros de digitação. Na descrição das variáveis categóricas foram utilizadas frequências absolutas e relativas. Utilizou-se o teste de Qui-quadrado para a verificação entre as proporções das variáveis: sexo, faixas etárias, dias da semana, dias do mês e classificações de risco. Admitiu-se um nível de significância estatística de 0,05. Realizaram-se os procedimentos de análise estatística com auxílio do software $R$.

O estudo foi desenvolvido em conformidade as diretrizes propostas pelas Resoluções 466/2012 e 510/16, e obteve aprovação do Comitê Permanente de Ética em Pesquisa com Seres Humanos da instituição (Parecer no 1.375.173). 


\section{RESULTADOS}

Dos atendimentos realizados pelas UPAs em novembro de 2015, constata-se que 2.927 foram ofertados a idosos, sendo 1.589 (54,3\%) na UPA 2 e 1.338 (46,6\%) pela UPA 1. A idade dos participantes variou de 60 a 103 anos, sendo quase a metade $(48,7 \%)$ do público atendido entre a faixa etária de 60 a 69 anos. As mulheres predominaram o grupo que mais procurou o serviço (55,7\%). O número de atendimento entre as UPAs nos dias da semana foi semelhante, sendo na UPA $1,69,5 \%$ de segunda a sexta-feira e 30,4\% no sábado e domingo, já na UPA 2 foi $65,4 \%$ e $34,5 \%$, respectivamente (Tabela 1 ).

TABELA 1: Caracterização sociodemográfica, dias de atendimento e classificação de risco dos atendimentos das UPAs. Maringá, PR, Brasil, 2016.

\begin{tabular}{|c|c|c|c|c|c|c|c|}
\hline \multirow{2}{*}{ Variáveis } & \multicolumn{2}{|c|}{ UPA 1} & \multicolumn{2}{|c|}{ UPA 2} & \multicolumn{2}{|c|}{ Total } & \multirow[t]{2}{*}{ Valor de $p^{*}$} \\
\hline & $\mathbf{N}$ & $\%$ & $\mathbf{N}$ & $\%$ & $\mathbf{N}$ & $\%$ & \\
\hline \multicolumn{8}{|l|}{ Sexo } \\
\hline Feminino & 758 & 56,6 & 875 & 55,0 & 1.633 & 55,7 & 0,410 \\
\hline Masculino & 580 & 43,3 & 714 & 44,9 & 1.294 & 44,2 & 0,430 \\
\hline \multicolumn{8}{|l|}{ Idade em anos } \\
\hline $60-69$ & 653 & 48,8 & 774 & 48,7 & 1.427 & 48,7 & 0,989 \\
\hline $70-79$ & 428 & 31,9 & 527 & 33,1 & 955 & 32,6 & 0,523 \\
\hline $80-89$ & 232 & 17,3 & 246 & 15,4 & 478 & 16,3 & 0,192 \\
\hline$>90$ & 25 & 1,8 & 42 & 2,6 & 67 & 2,2 & 0,203 \\
\hline \multicolumn{8}{|l|}{ Dias da semana } \\
\hline Dias úteis & 930 & 69,5 & 1.040 & 65,4 & 1970 & 67,3 & $0,021^{*}$ \\
\hline Final de semana & 408 & 30,4 & 549 & 34,5 & 957 & 32,6 & $0,021 *$ \\
\hline \multicolumn{8}{|l|}{ Classificação de } \\
\hline \multicolumn{8}{|l|}{ Risco } \\
\hline Azul & 415 & 31,0 & 158 & 9,9 & 573 & 19,5 & $<0,001^{*}$ \\
\hline Verde & 470 & 35,1 & 787 & 49,5 & 1.257 & 42,9 & $<0,001^{*}$ \\
\hline Amarelo & 380 & 28,4 & 622 & 39,1 & 1.002 & 34,2 & $<0,001^{*}$ \\
\hline Vermelho & 73 & 5,4 & 22 & 1,3 & 95 & 3,2 & $<0,001^{*}$ \\
\hline TOTAL & $\begin{array}{l}1.3 \\
38\end{array}$ & & 1.589 & & 2.927 & & \\
\hline
\end{tabular}

Nota: *Valor de $\mathrm{p}<0,05$ - teste Qui-quadrado.

Com relação as variáveis da classificação de risco, nota-se comportamento distinto entre as UPAs do município. Embora os números tenham se sobrepostos, em ambos os serviços predominou a classificação verde (casos pouco urgentes) com 46,5\% na UPA 2 e 35,1\% na UPA 1. Entretanto, a segunda classificação mais utilizada pelos enfermeiros da UPA 2 foi a amarela (casos urgentes) (39,1\%) e, na UPA 1 foi a azul (casos não urgentes) (26,1\%). Quanto às demais classificações, na UPA 2 seguiu-se com azul (9,9\%) e por último, vermelho (1,3\%). E, na UPA 1, 23,9\% dos idosos atendidos foram classificados como amarelo e 4,5\% como vermelho (Tabela 1).

Em relação as estratificações de idade associadas às classificações de risco, verifica-se diferenças significativas entre as faixas etárias de 60 a 69, e 80 a 89 anos nas UPAs estudadas (Tabela 2).

TABELA 2: Distribuição da classificação de risco por faixa etária das UPAs de um município paranaense. Maringá, PR, Brasil, 2016.

\begin{tabular}{|c|c|c|c|c|c|c|c|c|c|c|c|c|c|c|c|c|c|}
\hline \multirow{4}{*}{$\begin{array}{l}\text { Faixa } \\
\text { etária }\end{array}$} & \multicolumn{17}{|c|}{ Classificação de Risco } \\
\hline & \multicolumn{4}{|c|}{ Azul } & \multicolumn{4}{|c|}{ Verde } & \multicolumn{4}{|c|}{ Amarelo } & \multicolumn{4}{|c|}{ Vermelho } & \multirow[b]{3}{*}{ p-valor* } \\
\hline & \multicolumn{2}{|c|}{ UPA 1} & \multicolumn{2}{|c|}{ UPA 2} & \multicolumn{2}{|c|}{ UPA 1} & \multicolumn{2}{|c|}{ UPA 2} & \multicolumn{2}{|c|}{ UPA 1} & \multicolumn{2}{|c|}{ UPA 2} & \multicolumn{2}{|c|}{ UPA 1} & \multicolumn{2}{|c|}{ UPA 2} & \\
\hline & $\mathbf{N}$ & $\%$ & $\mathbf{N}$ & $\%$ & $\mathbf{N}$ & $\%$ & $\mathbf{N}$ & $\%$ & $\mathbf{N}$ & $\%$ & $\mathbf{N}$ & $\%$ & $\mathbf{N}$ & $\%$ & $\mathbf{N}$ & $\%$ & \\
\hline $60-69$ & 223 & 16,6 & 76 & 4,7 & 224 & 16,7 & 418 & 26,3 & 186 & 13,9 & 273 & 17,1 & 20 & 1,4 & 7 & 0,4 & $<0,001$ \\
\hline $70-79$ & 112 & 8,3 & 57 & 3,5 & 161 & 12 & 262 & 16,4 & 123 & 9,1 & 199 & 12,5 & 32 & 2,3 & 9 & 0,6 & 0,083 \\
\hline $80-89$ & 77 & 5,7 & 21 & 1,3 & 73 & 5,4 & 92 & 5,7 & 63 & 4,7 & 128 & 8 & 19 & 1,4 & 5 & 0,3 & $<0,001$ \\
\hline $90-99$ & 3 & 0,2 & 4 & 0,2 & 12 & 0,8 & 15 & 0,9 & 8 & 0,5 & 2 & 0,1 & 2 & 0,1 & 1 & 0,06 & 0,232 \\
\hline$\geq 100$ & 0 & 0 & 0 & 0 & 0 & 0 & 0 & 0 & 0 & 0 & 1 & 0,06 & 0 & 0 & 0 & 0 & 0,812 \\
\hline Total & 415 & 31 & 158 & 9,9 & 470 & 35,1 & 787 & 49,5 & 380 & 28,4 & 622 & 39,1 & 73 & 5,4 & 22 & 1,3 & \\
\hline
\end{tabular}

*Valor de $p<0,05$ - teste Qui-quadrado. 
Os dias da semana apresentaram distribuição equilibrada no número de atendimento entre as UPAs 1 e 2 , a qual percebe-se que segunda-feira houve mais atendimentos, 241 e 286 (527) seguida do domingo 220 e 283 (503) e, do sábado 188 e 239 (427) (Figura 1).

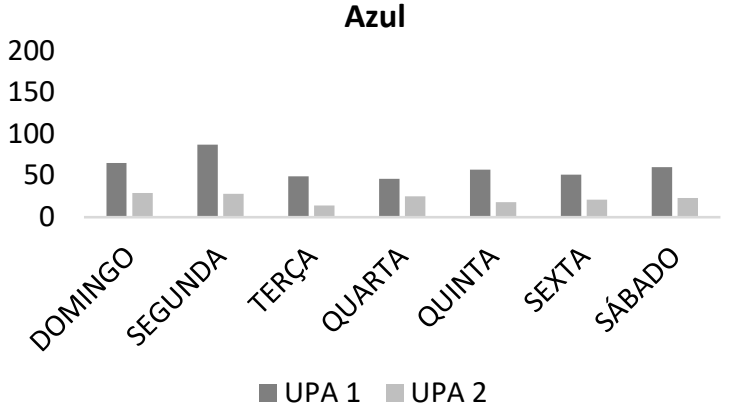

Amarelo

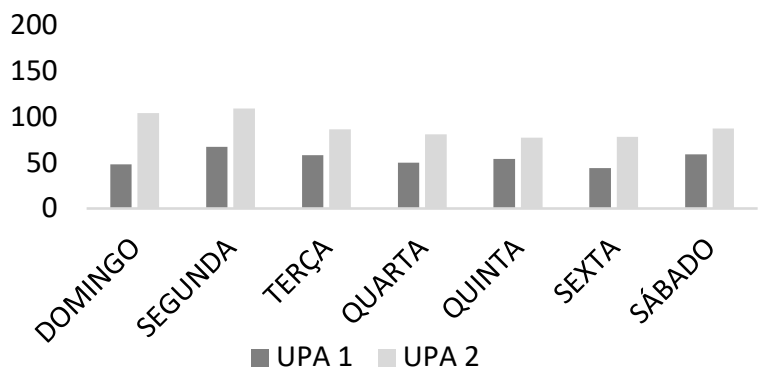

Verde

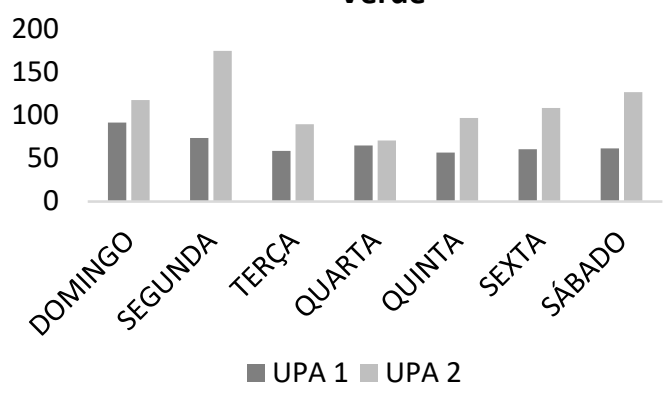

Vermelho

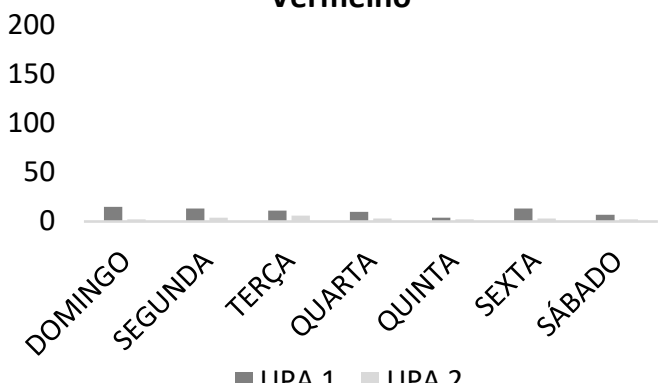

FIGURA 1: Distribuição dos atendimentos em conformidade à classificação de risco pelo dia da semana nas UPAs, Maringá, PR, Brasil, 2016.

Para às classificações de risco, observa-se que os idosos atendidos na UPA 1 foram mais classificados por azul na segunda-feira, seguida de domingo. Já na UPA 2, houve o inverso, o maior número de classificados por azul foi no domingo, seguido da segunda-feira. Quanto a coloração verde, na UPA 1 houve mais atendimentos no domingo enquanto na UPA 2 foram às segundas feira. Sobre a classificação amarela, em ambas as unidades foi na segunda-feira. E, a classificação vermelha foi mais utilizada aos domingos na UPA 1 e às terças-feiras pela UPA 2 (Figura 1).

Com relação aos atendimentos realizados nos dias do mês, a UPA 2 ofereceu número maior em quase todo o mês, com um pico no dia 21. Na UPA 1, os dias de maior atendimento foram 11, 18 e 25, com baixo número nos dias 04,12 , 20 e 24 (Figura 2).

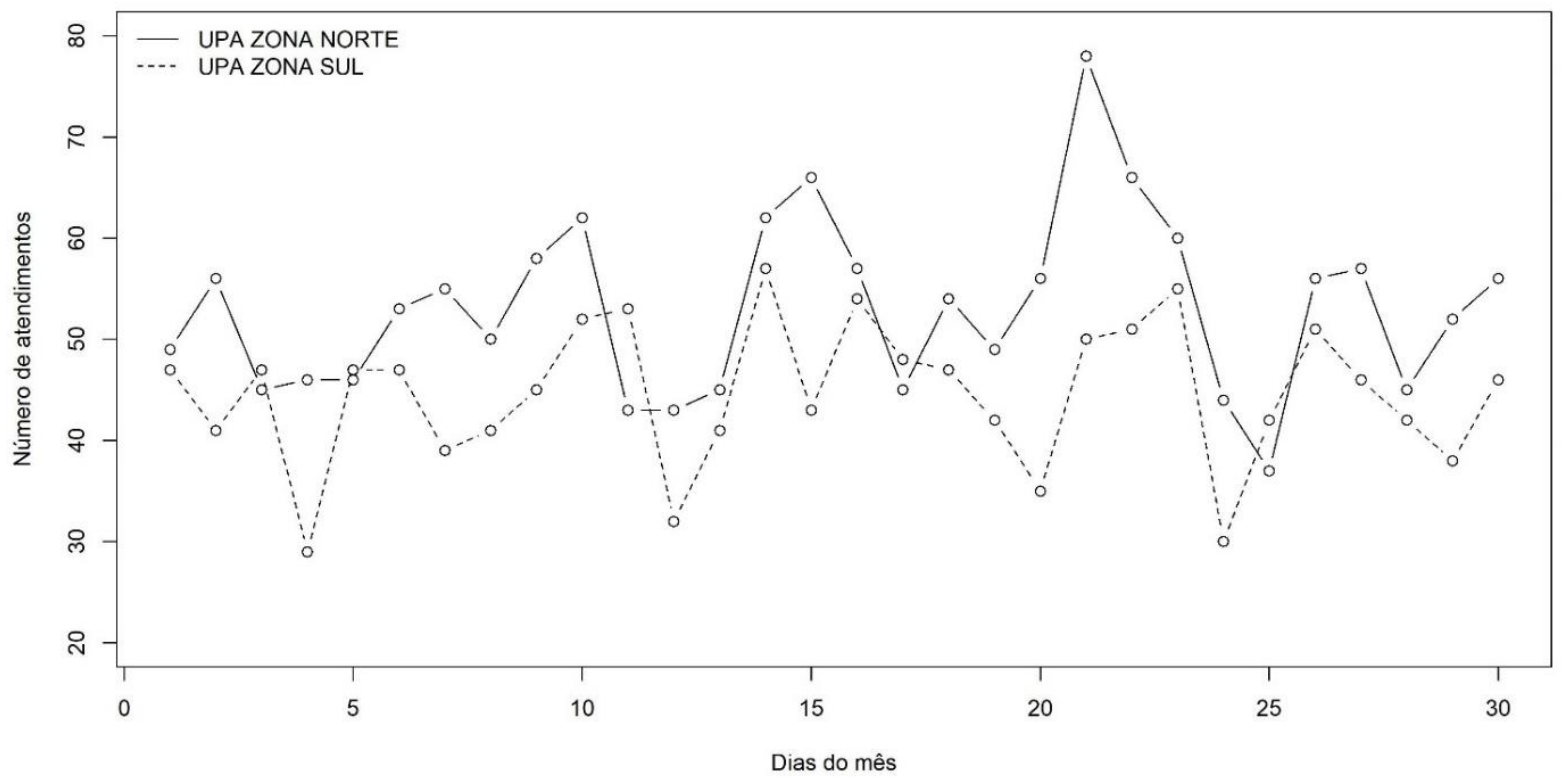

FIGURA 2: Atendimentos diários realizados nas UPAs, Maringá, PR, Brasil, 2016. 


\section{DISCUSSÃO}

A superlotação nos serviços emergenciais nos últimos anos fez com que o acolhimento com classificação de risco se tornasse uma ferramenta primordial nestes pontos da Rede de Atenção à Saúde (RAS). O presente estudo possibilitou verificar a relação da classificação de risco com as variáveis sexo, faixa etária, dias da semana e do mês e entre UPAs de um município paranaense. Os resultados demonstram necessidade em padronizar o acolhimento com classificação de risco e a importância emergente e efetiva de implementação das funções da Atenção Primária à Saúde (APS), a fim de consolidar o Sistema Único de Saúde (SUS) e oferecer assistência qualificada aos idosos.

Nota-se predominância de atendimento às mulheres, sendo que as UPAs 1 e 2 atenderam, 56\% e 55\% respectivamente. Este resultado é similar ao encontrado em pesquisa realizada em UPA de Minas Gerais, em que 57\% dos pacientes estudados eram do sexo feminino ${ }^{5}$. Estudos apontam que as mulheres, de modo geral, procuram mais os serviços de saúde quando comparado aos homens ${ }^{10,11}$ esse fenômeno pode estar relacionado a preocupação deste público sobre cuidados com a saúde ${ }^{12}$. Além disso há o fato da feminização da velhice, devido a fatores como à sobremortalidade masculina ${ }^{13}$, e o município estudado conta com $56,5 \%$ de mulheres idosas ${ }^{14}$.

A faixa etária que mais utilizou as UPAs foi de indivíduos entre 60 e 69 anos. Este achado se repete em vários estudos, indicando a predominância de pessoas com idade produtiva que buscam atendimentos em emergências $6,10,15$. Uma das condições que justificam a elevada procura de idosos jovens no serviço emergencial de média complexidade é a manutenção de suas atividades laborais, o que dificulta a procura pela APS, devido aos horários de atendimento e aspectos burocráticos deste nível de atenção ${ }^{4}$.

Por outro lado, a participação no mercado de trabalho e maior circulação no perímetro urbano, contribuem para ampliar as exposições à fatores de risco, como acidentes de trânsito e traumas, responsável pelo alto motivo de acesso ao sistema de saúde pelo atendimento pré-hospitalar móvel entre homens de 60 a 69 anos ${ }^{16}$. A qual trata-se de um serviço que intermedia o acesso dos usuários até os serviços de saúde, sobretudo das UPAs, que possuem porta aberta para a prestação de cuidados de complexidade intermediária.

O fato de ter mais idosos jovens nas UPAs indica também a agudizações de suas condições crônicas com maior frequência. Uma pesquisa realizada para identificar os motivos que levavam idosos com Condições Sensíveis à Atenção Primária (CSAP) a buscarem o serviço de uma UPA, identificou que 49,6\% eram idosos jovens (60 a 69 anos) e as principais queixas estavam relacionadas a doenças do sistema osteomuscular e tecido conjuntivo, do aparelho respiratório e circulatório 4 .

Associa-se o potencial uso das unidades de emergência a impossibilidade de resolução destes problemas em serviços da APS, o que está relacionado à utilização da Classificação de Risco de Manchester, por caracterizar os casos como "não urgentes" ou "pouco urgentes"10. Observa-se que em ambas UPAs, no presente estudo, sobressaíram o atendimento de idosos com situações clínicas que recebem as classificações azul e verde da CSAP, com 66,1\% na UPA 1 e 59,4\% na UPA 2. Os resultados também apontam as fragilidades da APS em executar suas funções de ordenadora e coordenadora do cuidado, o que provoca fragmentação da assistência e gera grandes desafios a todos os pontos da RAS.

Tal fato tem preocupado gestores e autoridades a nível mundial. Na literatura são encontradas experiências de outros países para minimizar as dificuldades entre os pontos da RAS, e o desenvolvimento de políticas com objetivo de diminuir a procura pelos serviços de atendimento à emergência, por onerar custos desnecessários ao sistema de saúde ${ }^{10}$. A exemplo, nos Estados Unidos da América, foi realizado um estudo de intervenção para integrar usuários atendidos em pronto-socorros aos serviços da APS ${ }^{16}$.

Sabe-se ainda que as alavancas para reduzir a busca desnecessária pela emergência também estão nos níveis dos determinantes sociais mais amplos de saúde, como renda, emprego, educação moradia e níveis de criminalidade ${ }^{10}$.

Os resultados do estudo apontam discrepâncias entre as UPAs estudadas. Em ambas, houve predomínio da classificação verde, sendo na UPA 1, 35,1\% e na UPA 2, 49,5\% dos atendimentos. Entretanto, ao comparar as demais classificações, constata-se que na UPA 2 os idosos foram classificados por amarelo (39,1\%), azul (9,9\%) e vermelho (5,4\%). Enquanto na UPA 1 foram classificados por azul (31\%), amarelo $(28,4 \%)$ e vermelho $(5,4 \%)$, respectivamente.

O sistema de triagem permite que o enfermeiro devidamente treinado, atribua prioridade clínica aos pacientes a partir da apresentação de seus sinais e sintomas ${ }^{17}$. Embora, a maioria dos enfermeiros de serviços emergenciais recebam preparo do protocolo de triagem institucional, nem sempre a classificação de risco ocorre de forma padronizada. Dentre as justificativas, estão as divergências de opiniões, não somente entre enfermeiros, mas também entre outros membros da equipe; e também, a ocorrência de subclassificações e superclassificação de gravidade ${ }^{17}$.

Reconhecendo as particularidades fisiológicas do envelhecimento, permite-se afirmar que o atendimento agudo na emergência é cada vez mais complexo, sendo que a avaliação e o gerenciamento de idosos nestes serviços consomem mais tempo e exigem habilidades especializadas ${ }^{18,19}$. Assim, julga-se emergente a necessidade de implementação de 
ações de educação permanente para padronização na classificação de risco, a fim de diminuir o viés da subjetividade afixado a funcionalidade e ao processo de decisão clínica na assistência a idosos ${ }^{20}$.

A relação entre as classificações de risco aos dias da semana demonstra que a coloração verde foi mais atribuída aos domingos pelos enfermeiros de ambas UPAs. Este achado demonstra que por tratar-se de um público que apresenta complicações agudizadas, sua condição clínica não permite aguardar até um dia útil da semana, em que há funcionamento dos serviços da APS.

Destaca-se que o maior número de atendimentos, somando todas as classificações de risco ocorreram às segundas-feiras nas duas Unidades. Dado que corrobora com pesquisa demonstrando que há duas vezes mais chances de procura dos serviços de emergência em dias úteis, estabelecendo, por vezes, o uso impróprio da UPA ${ }^{21}$. Assim como outros estudos, aponta a desarticulação da rede de atenção, principalmente pelo acesso irregular da população aos demais serviços, e a falta de resolutividade da APS ${ }^{10,22,23}$.

Referente a procura dos idosos pelos serviços de emergência ao longo do mês, vale destacar que apesar da UPA 1 ser classificada como porte III pelo Ministério da Saúde, na UPA 2 houve mais atendimentos. A UPA 1 sobrepôs o quantitativo de atendimentos da UPA 2 somente em três datas, 11, 18 e 25. Trata-se de um dado considerável, pois as UPAs recebem financiamentos e oferecem recursos de acordo com a classificação de seu porte. Houve maior número de atendimentos na Unidade de menor porte, apontando necessidade de revisão da gestão local e municipal, na investigação das possíveis diferenças e intervenções nas dificuldades enfrentadas para a assistência dos usuários nestes serviços.

\section{Limitações do estudo}

Como limitações, o estudo utilizou dados secundários referentes ao registro no banco de dados, o qual depende das informações registradas pelos profissionais da Unidade, e que podem interferir na qualidade destas. Outro fato é o estudo retratar as Unidades de um município, o que pode limitar a generalização dos achados. Entretanto, os resultados proporcionam uma perspectiva de análise para aplicação em outras realidades, o que representa uma das recomendações para novos estudos.

\section{CONCLUSÃO}

O acolhimento de idosos entre as UPAs, segundo as categorias da classificação de risco com as variáveis estudadas, demonstrou que os idosos jovens têm apresentado frequente agudizações das condições crônicas, levando-os a procurar por este nível de atenção, sobretudo por CSAP e às segundas-feiras. Esta característica representa uma fragmentação da Rede de Atenção, com comprometimento de todo o sistema de saúde.

A partir deste estudo foi possível identificar importante discrepância entre o número de atendimentos e as classificações de risco das Unidades. Tais achados poderão auxiliar no direcionamento das capacitações para a padronização do acolhimento com classificação de risco, além de contribuir para o aprimoramento das funções da APS, no intuito de oferecer assistência integral e longitudinal aos idosos, propiciando qualidade de vida e ao mesmo tempo, consolidação do SUS.

Os resultados ainda podem contribuir com a gestão local na organização da RAS, por conter dados importantes para avaliar e reorganizar este ponto de atenção no município, indicando a importância desse tipo de estudo ser replicado em outras localidades como suporte avaliativo e de gerência do sistema de saúde.

\section{REFERÊNCIAS}

1. Chatterji S, Byles J, Cutler D, Seeman T, Verdes E. Health, functioning, and disability in older adults - present status and future implications. The Lancet [Internet]. 2015 [cited 2017 Mar 24]; 385. DOI: https://doi.org/10.1016/s0140-6736(14)61462-8.

2. Bloom DE et al. Macroeconomic implications of population ageing and selected policy responses. The Lancet [Internet]. 2015 [cited 2018 Mar 24]; 385:649-57. DOI: https://doi.org/10.1016/s0140-6736(14)61464-1.

3. Franchi C, Cartabia M, Santalucia P, Baviera M, Mannucci PM, Fortino I, et al. Emergency department visits in older people: pattern of use, contributing factors, geographical differences and outcome. Aging Clin. Exp. Res. [Internet]. 2017 [cited 2018 Mar 24]; 29(2):319-26. DOI: https://doi.org/10.1007/s40520-016-0550-5.

4. Rissardo LK, Rego AS, Scolari GAS, Radovanovic CAT, Decesaro MN, Carreira L. Elderly care unit ready for sensitive conditions to primary health care. Reme, Rev. Min. Enferm. [Internet]. 2016 [cited 2018 Mar 24]; 20(e971)1-8. DOI: http://dx.doi.org/10.5935/1415-2762.20160041.

5. Diniz AS, Silva AP, Souza CC, Chianca TCM. Clinical demand in an emergency care unit according to the Manchester triage system. Rev. Eletr. Enf. [Internet]. 2014 [cited 2018 Mar 19]; 16(2):312-20. DOI: http://dx.doi.org/10.5216/ree.v16i2.21700.

6. Antunes BCS, Crozeta K, Assis F, Paganini MC. Urgent and emergency care network: profile, demand and flow of elderly to health care points. Cogitare Enferm. [Internet]. 2018 [cited 2018 Mar 19]; 23(2):e53766. DOI: http://dx.doi.org/10.5380/ce.v23i2.53766. 
7. Ministério da Saúde (Br), Secretaria de Assistência à Saúde. Programa Nacional de Humanização da Assistência Hospitalar. Brasília: Ministério da Saúde, p. 60, 2006.

8. Oliveira TA, Pinto KA. Welcome with risk classification and the conditions of access in emergency service: users' evaluation. Cienc., Cuid. Saúde. [Internet]. 2015 [cited 2018 Mar 19]; 14(2):1122-9. DOI: https://doi.org/10.4025/cienccuidsaude.v14i2.22897.

9. Instituto Paranaense de Desenvolvimento Econômico e Social. IPARDES. Caderno Estatístico do Município de Maringá [Internet], Curitiba (BR): [sem editor]; 2019; [cited 2018 Mar 18].

10. Schafirowitz GC, Souza AC. Adult users classified as least urgent in Emergency Care Unit. Interface (Botucatu) [Internet]. 2020 [cited 2018 Mar 19]; 24(Supl. 1):e190630. DOI: https://doi.org/10.1590/Interface.190630.

11. Malta DC, Bernal RTI, Lima MG, Araújo SSC, Silva MMA, Freitas MIF, Barros MBA. Noncommunicable diseases and the use of health services: analysis of the National Health Survey in Brazil. Rev. Saude Publica [Internet]. 2017 [cited 2018 Mar 19]; 51(Supl. 1):4s. DOI: https://doi.org/10.1590/S1518-8787.2017051000090.

12. Antunes BCS, Crozeta K, Assis F, Paganini MC. Urgent and emergency care network: profile, demand and flow of elderly to health care points. Cogitare Enferm. [Internet]. 2018 [cited 2018 Mar 19]; 23(2):e53766. DOI: http://dx.doi.org/10.5380/ce.v23i2.53766.

13. Mincoff RCL, Bennemann RM, Martins MC. Estado nutricional de idosos participantes do sistema Hiperdia: características sociodemográficas e níveis pressóricos. Rev. Rene [Internet]. 2015 [cited 2018 Mar 19]; 16(5):746-53. DOI: https://doi.org/10.15253/2175-6783.2015000500017.

14. Instituto Brasileiro de Geografia e Estatística. IBGE. Censo demográfico de 2010: dados preliminares do universo. [Internet], Brasília, DF: MDS; 2011 [cited 2016 Apr 29]. Available from: https://www.ibge.gov.br/home/estatistica/populacao/censo2010/default_resultados_universo.shtm.

15. Dixe MACR, Passadouro R, Peralta TFC, Lourenço GS, Pedro ML. Determinants of non-urgent emergency department use. Rev. Enf. Ref. [Internet]. 2018 [cited 2019 Jun 7]; 4(16):41-52. DOI: https://doi.org/10.12707/RIV17095.

16. Wexler R, Hefner JL, Sieck C, Taylor CA, Lehman J, Panchal AR, Aldrich A, McAlearney AS. Connecting Emergency Department Patients to Primary Care. J. Am. Board. Fam. Med. [Internet]. 2015 [cited 2019 Jun 7]; 28(6):722-32. DOI: https://doi.org/10.3122/jabfm.2015.06.150044.

17. Herminda PMV, Jung W, Nascimento ERP, Silveira NR, Alves DLF, Benfatto TB. Risk classification in an emergency care unit: the nurses' discourse. Rev. Enferm. UERJ. [Internet]. 2017 [cited 2019 Jun 7]; 25:e19649. DOI: https://doi.org/10.12957/reuerj.2017.19649.

18. Ellis G, Marshall T, Ritchie C. Comprehensive geriatric assessment in the emergency department. Clinical Interventions in Aging [Internet]. 2014 [cited 2019 Jun 7]; 9:2033-43. DOI: https://dx.doi.org/10.2147\%2FCIA.S29662.

19. McCabe JJ, Cournane S, Byrne D, Conway R, O'Riordan D, Silke B. Age and the economics of an emergency medical admissionwhat factors determine costs? QJM: An International Journal of Medicine [Internet]. 2017 [cited 2019 Jun 7]; 110(2):83-8. DOI: https://doi.org/10.1093/qjmed/hcw161.

20. Ferreira WFS, et al. Urgency and emergency in the dimensions of the elderly: Manchester system. Saúde e desenvolvimento [Internet]. 2017 [cited 2019 Jun 7];11(6):283-93. Available from: https://www.revistasuninter.com/revistasaude/index.php/saudeDesenvolvimento/article/view/637.

21. Machado GVC, Oliveira FLP, Barbosa HAL, Giatti LBPF. Associated factors to the use of an emergency service, Ouro Preto, 2012. Cad. Saude Colet. [Internet]. 2015 [cited 2019 Jun 7]; 23(4):416-24. DOI: http://dx.doi.org/10.1590/1414-462X201500040177.

22. Rêgo AS, Rissardo LK, Scolari GAS, Sanches RCN, Carreira L, Radovanovic CAT. Factors associated with the care of elderly persons with Primary Health Care sensitive conditions. Rev. Bras. Geriatr. Gerontol. [Internet], 2017 [cited 2019 Jun 7]; 20(6):773-84. DOI: https://doi.org/10.1590/1981-22562017020.170120.

23. Cassettari SSR, Mello ALSF. Demand and type of care provided in emergency services in the city of Florianópolis, Brazil. Texto contexto Enferm. [Internet], 2017 [cites 2019 Jun 7]; 26(1):e3400015. DOI: https://doi.org/10.1590/0104-07072017003400015. 